\title{
Decreased Bleeding Rates in Patients with Hemophilia A Switching from Standard-Half-Life FVIII to BAY 94-9027 Prophylaxis
}

\author{
Maria Elisa Mancuso ${ }^{10}$ Mark T. Reding ${ }^{2}$ Claude Negrier $^{3} \quad$ Bryce A. Kerlin $^{4}$ Savita Rangarajan ${ }^{5}$ \\ Mindy L. Simpson ${ }^{6}$
}

${ }^{1}$ Center for Thrombosis and Hemorrhagic Diseases, Humanitas Clinical and Research Center-IRCCS, Rozzano, Milan, Italy

Address for correspondence Maria Elisa Mancuso, MD, PhD, Center for Thrombosis and Hemorrhagic Diseases, Humanitas Clinical and Research Center-IRCCS, Via Manzoni 56, 20089, Rozzano, Milan, Italy

2 University of Minnesota Medical Center, Minneapolis, Minnesota, United States

${ }^{3}$ Louis Pradel University Hospital, University Claude Bernard Lyon 1, (e-mail: mariaelisa_mancuso@libero.it; elisamancuso@gmail.com).

Lyon, France

${ }^{4}$ Nationwide Children's Hospital and The Ohio State University

College of Medicine, Columbus, Ohio, United States

${ }^{5}$ University Hospital Southampton NHS Foundation Trust,

Southampton, United Kingdom

${ }^{6}$ Rush University Medical Center, Chicago, Illinois, United States

Thromb Haemost 2021;121:1079-1086.

\begin{abstract}
Keywords

- annualized bleeding rate

- hemophilia A

- prophylaxis

- recombinant factor VIII

- target joint

BAY 94-9027 (damoctocog alfa pegol, Jivi) is an extended-half-life recombinant factor VIII (rFVIII) shown to be well-tolerated and efficacious in bleeding prevention in previously treated patients with severe hemophilia A. During the PROTECT VIII study, prophylaxis patients received BAY 94-9027 at intervals determined based on their bleeding phenotype, observed during a 10 -week run-in treatment period with twiceweekly dosing. Those with $\leq 1$ spontaneous joint or muscle bleed were randomized to either 45 to $60 \mathrm{IU} / \mathrm{kg}$ every 5 days or $60 \mathrm{IU} / \mathrm{kg}$ every 7 days; patients could increase dosing frequency to every 5 days or twice weekly in the case of bleeds. Those enrolled after the randomization arms were full, and those with $\geq 2$ bleeds in the run-in period, received 30 to $40 \mathrm{lU} / \mathrm{kg}$ twice weekly. Patients completing the main study could receive open-label BAY 94-9027 in the extension phase. Dosing regimen, total, and joint annualized bleeding rates were analyzed over three periods: prestudy, main study, and extension. A total of 80 patients who were on prophylaxis treatment prior to and during the study and had prior bleed data available were evaluated in this post hoc analysis of PROTECT VIII. Most patients (> 80\%) required fewer infusions with BAY 94-9027 prophylaxis versus their previous standard-half-life (SHL) rFVIII product. Lower bleeding and joint bleeding rates were observed over time from the prestudy to the extension study period in all treatment regimens. Compared with SHL FVIII, BAY 94-9027 prophylaxis allows patients to reduce infusion frequency with maintained or improved protection from bleeds.
\end{abstract}

received

May 7, 2020

accepted after revision

December 8, 2020

published online

December 9, 2020
DOI https://doi.org/ $10.1055 / \mathrm{a}-1333-5536$ ISSN $0340-6245$. (c) 2020. The Author(s).

This is an open access article published by Thieme under the terms of the Creative Commons Attribution-NonDerivative-NonCommercial-License, permitting copying and reproduction so long as the original work is given appropriate credit. Contents may not be used for commercial purposes, or adapted, remixed, transformed or built upon. (https://creativecommons.org/ licenses/by-nc-nd/4.0/)

Georg Thieme Verlag KG, Rüdigerstraße 14, 70469 Stuttgart, Germany 


\section{Introduction}

Prophylaxis with factor VIII (FVIII) concentrates is the current standard of care to prevent bleeding episodes in patients with severe hemophilia A. ${ }^{1}$ If initiated early enough, prophylaxis can decrease the frequency of joint hemorrhages and prevent ensuing joint damage, thereby halting the development of chronic hemophilic arthropathy. ${ }^{2,3}$ Patients receiving prophylactic treatment also miss fewer days from school or work, spend fewer days in hospital, and have a better quality of life compared with those treated on demand. ${ }^{4}$ However, standardhalf-life (SHL) FVIII products, which include plasma-derived FVIII and recombinant FVIII (rFVIII) products, have short halflives, and hence require frequent intravenous infusions. ${ }^{5,6}$ Effective prophylaxis with standard FVIII products usually requires infusions to be administered three times per week or every other day, representing a significant treatment burden for patients and caregivers. ${ }^{5}$ Consequently, adherence to prophylactic treatment is difficult to be maintained in these patients, ${ }^{7,8}$ hence increasing the risk of breakthrough bleeds, including joint bleeds. ${ }^{9}$ Extended-half-life (EHL) products are specifically designed to prolong the time in circulation and the coagulation factor half-life. In the case of EHL FVIII, the FVIII protein has undergone structural modifications, such as PEGylation, fusion protein technology, or single chain technology. EHL coagulation factors aim to decrease the dose frequency by maintaining high trough levels, without compromising on safety and efficacy. ${ }^{10}$

BAY 94-9027 (damoctocog alfa pegol, Jivi) is an EHL, Bdomain-deleted rFVIII that is site-specifically PEGylated with a single $60 \mathrm{kDa}$ (30 kDa dual-branched) polyethylene glycol molecule to improve its pharmacokinetics. ${ }^{11}$ In a phase 1 study in previously treated patients with severe hemophilia A, BAY 94-9027 demonstrated decreased clearance, greater area under the curve (AUC), and a longer half-life as compared with SHL, sucrose-formulated rFVIII (rFVIII-FS [Kogenate FS, Bayer, Berkeley, California, United States]). ${ }^{12}$ The efficacy and safety of BAY 94-9027 as prophylactic and ondemand treatment for patients with hemophilia A were demonstrated in the multinational phase 2/3 PROTECT VIII study and its long-term extension. ${ }^{13-15}$ BAY 94-9027 has been approved by the U.S. Food and Drug Administration (FDA), the European Medicines Agency (EMA), Health Canada, and the Pharmaceuticals and Medical Devices Agency (PMDA) in Japan for use in previously treated adults and adolescents (aged $\geq 12$ years) with hemophilia $A$ at dosing intervals of up to every 5 days (FDA, Canada) and every 7 days (EMA and PMDA). ${ }^{16-18}$

Although a matched adjusted indirect comparison between BAY 94-9027 and other EHL rFVIIIs (efmoroctocog alfa [rFVIII-Fc, Eloctate; Sanofi Genzyme] and rurioctocog alfa pegol [BAX 855, Adynovate; Takeda]) and a SHL rFVIII (rAHF-PFM [Baxter]) suggested that BAY 94-9027 utilization was lower than for these other rFVIII products, with comparable bleed protection, ${ }^{19}$ there is a lack of intrapatient comparisons of bleed rates and dosing frequency for SHL versus EHL FVIII products used as prophylaxis. Here, we report the findings of a post hoc analysis of the PROTECT VIII data comparing bleeding rates for patients treated with BAY 94-9027 prophylaxis with those for their previous SHL-FVIII prophylactic treatment.

\section{Methods}

\section{Study Design}

The design of the PROTECT VIII trial (Clinicaltrials.gov identifier, NCT01580293) has been previously described. ${ }^{13}$ Briefly, male patients aged 12 to 65 years with severe hemophilia A (FVIII $<1 \%)$, previously treated with any FVIII product for $\geq$ 150 exposure days (EDs), were eligible. Written informed consent was provided by all patients or their legal guardians before entry into the study, and the study protocol was approved by each study center's independent ethics committee/institutional review board.

PROTECT VIII was a partially randomized, open-label, phase 2/3 study. Patients received BAY 94-9027 for 36 weeks either on demand $(n=20)$ or prophylactically $(n=114)$. Patients receiving prophylaxis completed a 10 -week run-in period, during which they received BAY 94-9027 $25 \mathrm{IU} / \mathrm{kg}$ twice weekly.

Patients who experienced $\geq 2$ spontaneous bleeds (defined as joint or muscle bleeds with no identified trauma) during this period, maintained prophylaxis with 30 to 40 $\mathrm{IU} / \mathrm{kg}$ twice weekly until study completion. Patients with $\leq 1$ breakthrough bleed during the run-in period were randomized 1:1 to receive BAY 94-9027 either 45 to $60 \mathrm{IU} / \mathrm{kg}$ every 5 days or $60 \mathrm{IU} / \mathrm{kg}$ every 7 days. Patients could increase their dosing frequency to every 5 days or twice weekly in case of bleeds. Additional patients enrolled when the randomization arms were already full continued with BAY 94-9027 30 to $40 \mathrm{IU} / \mathrm{kg}$ twice weekly despite being eligible for randomization according to their bleeding tendency.

Patients completing the main study could enroll in an optional extension study. ${ }^{14}$ At the start of the extension, patients could continue their regimen from the main study, either on demand or prophylaxis, or switch to any of the three prophylaxis regimens. Patients who switched regimen later during the extension study, after the first 7 days of being in the extension, were analyzed in a combined variable frequency group.

This is a post hoc analysis of patients who received SHL FVIII prophylaxis prior to entry into the PROTECT VIII study, received any BAY 94-9027 prophylaxis regimen during the main study, and continued with prophylaxis into the extension study (data cutoff, August 2019). Patients were analyzed in the prophylaxis regimens of the extension study, independent of the regimen in the main study. Only patients with information available for prestudy FVIII prophylaxis treatment and prestudy 12-month bleeding rate were included. Patients who were treated on demand before, with prestudy data available, and during the main and extension studies were included as a reliability group.

\section{Efficacy Assessments}

During the PROTECT VIII study, bleeding events and administered infusions were recorded by patients using an 
electronic diary. Annualized bleeding rates (ABRs) were calculated for all bleeds and joint bleeds.

Prestudy data were self-reported by patients at the baseline visit and were recorded in an electronic case report form; these data included FVIII product, dosing regimen, and number of bleeding episodes during the 12 months prior to study entry. Prestudy treatment type (on demand vs. prophylaxis) and bleed information was based on documentation in medical records. The prestudy prophylaxis regimen was reported by the patients in the Treatment Satisfaction and Burden questionnaire, which was completed at baseline.

\section{Statistical Analysis}

Statistical analysis was performed using SAS software 9.2 (SAS Institute Inc., Cary, North Carolina, United States). Summary statistics were calculated for continuous data, and frequencies were calculated for categorical data. Dosing regimen and $A B R s$ were reported over three periods: prestudy, main study, and extension study. Prestudy treatment frequency was recorded in the patient questionnaire. As a reliability assessment of prestudy bleed data collected from medical records, ABRs were evaluated for patients treated with BAY 94-9027 on demand in whom no improvement in ABRs would be expected between the three periods. Patients were analyzed by regimen in the extension study. Patients who switched at least once to another regimen $\geq 7$ days since the start of the extension study were analyzed in the variable frequency group.

\section{Results}

\section{Study Population}

Of 126 patients who completed the PROTECT VIII main study (on-demand, $n=18$; prophylaxis, $n=108$ ), 121 patients chose to continue in the optional extension study, receiving either ondemand treatment $(n=14)$ or prophylaxis $(n=107)$. Out of a total of 82 patients who received prophylaxis prestudy and during the main study and extension, 80 were considered for this analysis; 14 patients received on-demand treatment during the three periods and were considered as a reliability group, included to assess the accuracy of pre- and poststudy reporting of bleeds ( - Fig. 1). Data on previous regimens and prior bleed frequency were available for 80 of the 82 patients receiving BAY 94-9027 prophylaxis during the PROTECT VIII main study and its extension. The remaining 25 of the 107 patients who continued into the extension on a prophylaxis regimen were treated ondemand prior to study entry and so were not included in the prophylaxis group of this analysis. Baseline demographics and disease characteristics were similar between the prophylactic treatment groups ( - Table 1 ). At baseline, $72.5 \%$ of patients had target joints, with a median of 1.0 target joint per patient, despite receiving prophylaxis prior to the study.

At data cutoff (August 2019), patients in the prophylaxis treatment arms had spent a median (range) of 3.9 (0.8-6.8) years in the combined main study and extension, with a median of 280 (57-677) EDs. Patients in the on-demand treatment arm had spent a median (range) of 3.9 (1.3-4.8)

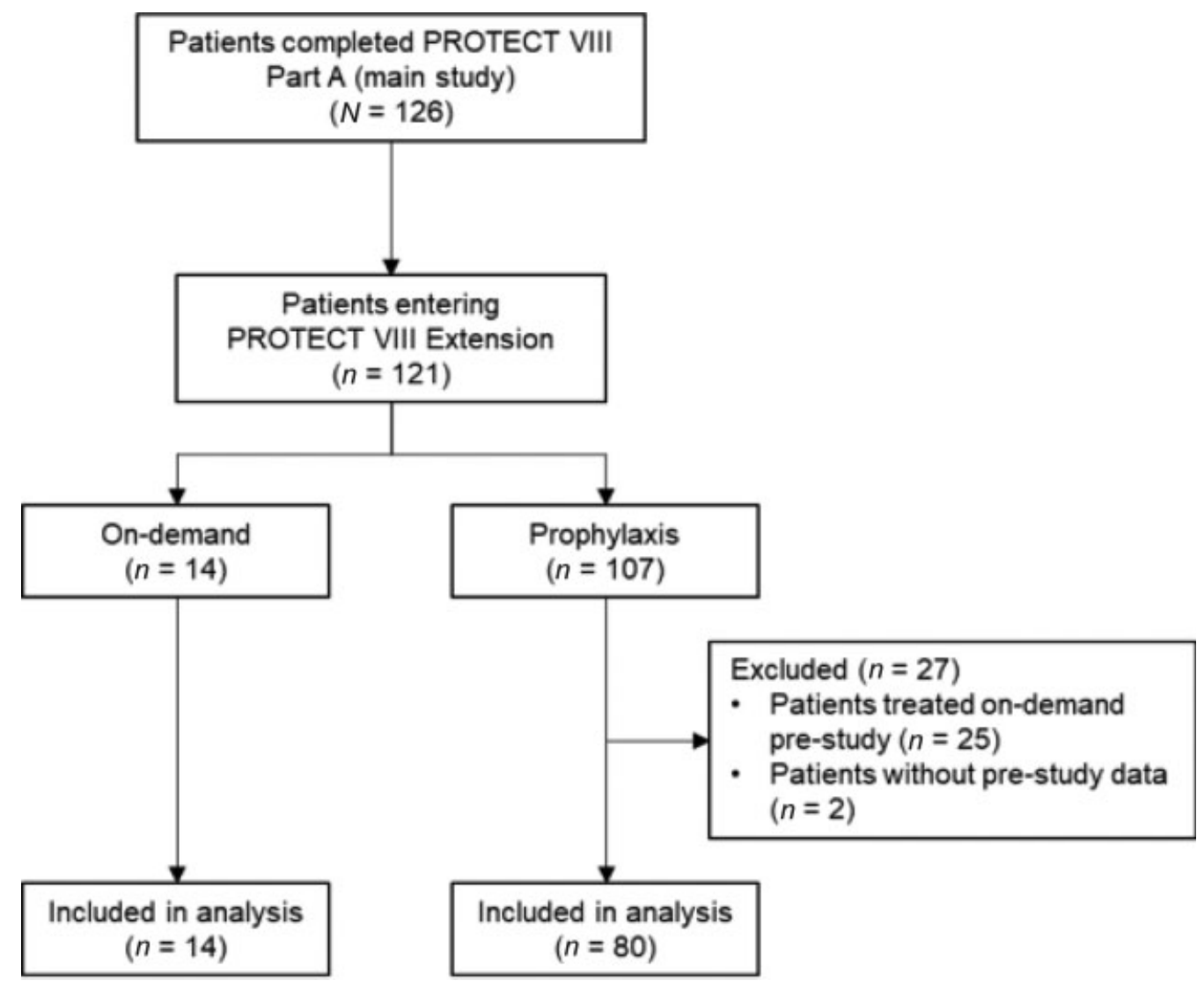

Fig. 1 Patient disposition. 
Table 1 Patient demographics and baseline disease characteristics

\begin{tabular}{|c|c|c|c|c|c|c|}
\hline & $\begin{array}{l}\text { On-demand } \\
(n=14)\end{array}$ & $\begin{array}{l}\text { Twice weekly } \\
(n=13)\end{array}$ & $\begin{array}{l}\text { Every } 5 \text { days } \\
(n=28)\end{array}$ & $\begin{array}{l}\text { Every } 7 \text { days } \\
(n=17)\end{array}$ & $\begin{array}{l}\text { Variable } \\
\text { frequency }^{a} \\
(n=22)\end{array}$ & $\begin{array}{l}\text { Total } \\
\text { prophylaxis } \\
(N=80)\end{array}$ \\
\hline $\begin{array}{l}\text { Age, y } \\
\text { Median (Q1; Q3) }\end{array}$ & $\begin{array}{l}43.5 \\
(29.0 ; 58.0)\end{array}$ & $\begin{array}{l}31.0 \\
(19.0 ; 41.0)\end{array}$ & $\begin{array}{l}34.5 \\
(24.5 ; 43.5)\end{array}$ & $\begin{array}{l}31.0 \\
(26.0 ; 52.0)\end{array}$ & $\begin{array}{l}37.0 \\
(20.0 ; 47.0)\end{array}$ & $\begin{array}{l}32.5 \\
(24.0 ; 46.0)\end{array}$ \\
\hline $\begin{array}{l}\text { Race, } n(\%) \\
\quad \text { White }\end{array}$ & $7(50.0)$ & $10(76.9)$ & $22(78.6)$ & $10(58.8)$ & $16(72.7)$ & $58(72.5)$ \\
\hline $\begin{array}{l}\text { BMI, } \mathrm{kg} / \mathrm{m}^{2} \\
\quad \text { Median (Q1; Q3) }\end{array}$ & $\begin{array}{l}26.0 \\
(21.4 ; 28.7)\end{array}$ & $\begin{array}{l}22.6 \\
(21.1,25.9)\end{array}$ & $\begin{array}{l}25.5 \\
(23.2,27.5)\end{array}$ & $\begin{array}{l}24.9 \\
(22.3,27.8)\end{array}$ & $\begin{array}{l}24.6 \\
(21.4,28.8)\end{array}$ & $\begin{array}{l}24.6 \\
(21.4,27.8)\end{array}$ \\
\hline $\begin{array}{l}\text { Patients with target } \\
\text { joints, } n(\%)\end{array}$ & $11(78.6)$ & $11(84.6)$ & 19 (67.9) & $10(58.8)$ & $18(81.8)$ & $58(72.5)$ \\
\hline $\begin{array}{l}\text { Number of target joints } \\
\text { per patient } \\
\text { Mean (SD) } \\
\text { Median (Q1; Q3) }\end{array}$ & $\begin{array}{l}2.7(2.3) \\
2.5(1.0 ; 4.0)\end{array}$ & $\begin{array}{l}1.4(0.9) \\
1.0(1.0,2.0)\end{array}$ & $\begin{array}{l}1.2(1.1) \\
1.0(0.0,2.0)\end{array}$ & $\begin{array}{l}1.0(1.1) \\
1.0(0.0,2.0)\end{array}$ & $\begin{array}{l}1.9(1.6) \\
1.5(1.0,2.0)\end{array}$ & $\begin{array}{l}1.4(1.2) \\
1.0(0.0,2.0)\end{array}$ \\
\hline \multicolumn{7}{|c|}{ Treatments per week before study, $n$ (\%) } \\
\hline 3 or 4 times per week & $1(7.1)$ & $9(69.2)$ & $16(57.1)$ & $9(52.9)$ & $15(68.2)$ & $49(61.3)$ \\
\hline 2 times per week & $1(7.1)$ & $4(30.8)$ & $9(32.1)$ & $7(41.2)$ & $7(31.8)$ & $27(33.8)$ \\
\hline 1 time per week & $1(7.1)$ & 0 & $1(3.6)$ & $1(5.9)$ & 0 & $2(2.5)$ \\
\hline $\begin{array}{l}\text { On demand, when } \\
\text { having a bleed }^{\mathrm{b}}\end{array}$ & $11(78.6)$ & 0 & $1(3.6)$ & 0 & $1(1.3)$ & \\
\hline Missing & 0 & $1(3.6)$ & 0 & \multicolumn{3}{|l|}{$1(1.3)$} \\
\hline
\end{tabular}

Abbreviations: BMI, body mass index; Q, quartile; SD, standard deviation. Note: Treatment groups based on regimen during the extension study.

apatients who changed treatment group at least once after first infusion beyond 7 days into extension.

${ }^{\mathrm{b}}$ As reported at patient's questionnaire. Preprophylaxis regimen was confirmed by investigator.

years in the combined main study and extension, with a median of 134 (23-222) EDs.

\section{Prophylactic Treatment Frequency}

Prior to the study, $n=49 / 80$ (61.3\%) received prophylactic treatment three or four times per week and 27/80 (33.8\%) had been treated twice weekly. Two patients $(2.5 \%)$ received treatment once weekly. Frequency of prestudy prophylaxis was unknown in the remaining two patients (-Fig. 2). Most ( $>80 \%$ ) patients decreased the frequency of prophylaxis infusions per week during the PROTECT VIII main study and its extension compared with the prestudy period. Of the 80 patients on a prophylaxis regimen during the extension at data cutoff, 12 were enrolled in the twice-weekly prophylaxis group in the main study, 32 were enrolled in the every-5-day prophylaxis group, and 36 were enrolled in the every-7-day prophylaxis group. By data cutoff, 22 of these patients were analyzed in the variable frequency group after switching their treatment regimen used in the main study during the extension phase (-Fig. 2 ).

\section{Annualized Bleeding Rate}

In patients who received prophylaxis prior to and during the PROTECT VIII study and its extension $(n=80)$, median (Q1; Q3) ABR for total bleeds was reduced in the extension (1.6 [0.4; $4.8])$ compared with the prestudy $(3.5[1.0 ; 10.0])$ and the main study $(2.1[0.0 ; 7.1])$ periods. During the extension, median total ABRs for twice-weekly, every-5-day, and every-7-day prophylaxis regimens were $2.3(1.0 ; 10.7), 1.2(0.1 ; 4.2)$, and
$0.6(0.0 ; 1.6)$, respectively. Median total ABR for patients in the variable frequency group was $3.1(1.1 ; 6.4)$ (-Fig. 3A).

\section{Joint ABR}

In patients who received prophylaxis prior to and during the PROTECT VIII study and its extension $(n=80)$, median (Q1; Q3) ABR for joint bleeds was reduced in the extension (1.0 $[0.0 ; 3.4])$ compared with the prestudy $(2.0[0.0 ; 8.0])$ and the main study $(1.4[0.0 ; 4.5])$ periods. During the extension, median joint ABRs for patients in the twice-weekly, every-5day, and every-7-day prophylaxis arms were $0.7(0.0 ; 1.7)$, $1.0(0.0 ; 3.4)$, and $0.4(0.0 ; 1.2)$, respectively. Median joint ABR for patients in the variable frequency group was 2.0 (0.6; 4.6) (-Fig. 3B). At the cutoff date, patients in the prophylaxis and on-demand treatment arms had median target joint ABRs of $0.0(0.0 ; 1.4)$ and $12.9(0.0 ; 27.3)$, respectively.

\section{Reliability Group}

Reliability of retrospective prestudy bleed rates was shown by a lack of improvement in ABRs and joint ABRs in patients previously treated on-demand who received BAY 94-9027 on demand $(n=14)$ during the main study and extension periods (-Fig. 3 ).

\section{Discussion}

This post hoc analysis directly compared the prophylactic infusion frequency and bleeding rates observed when 


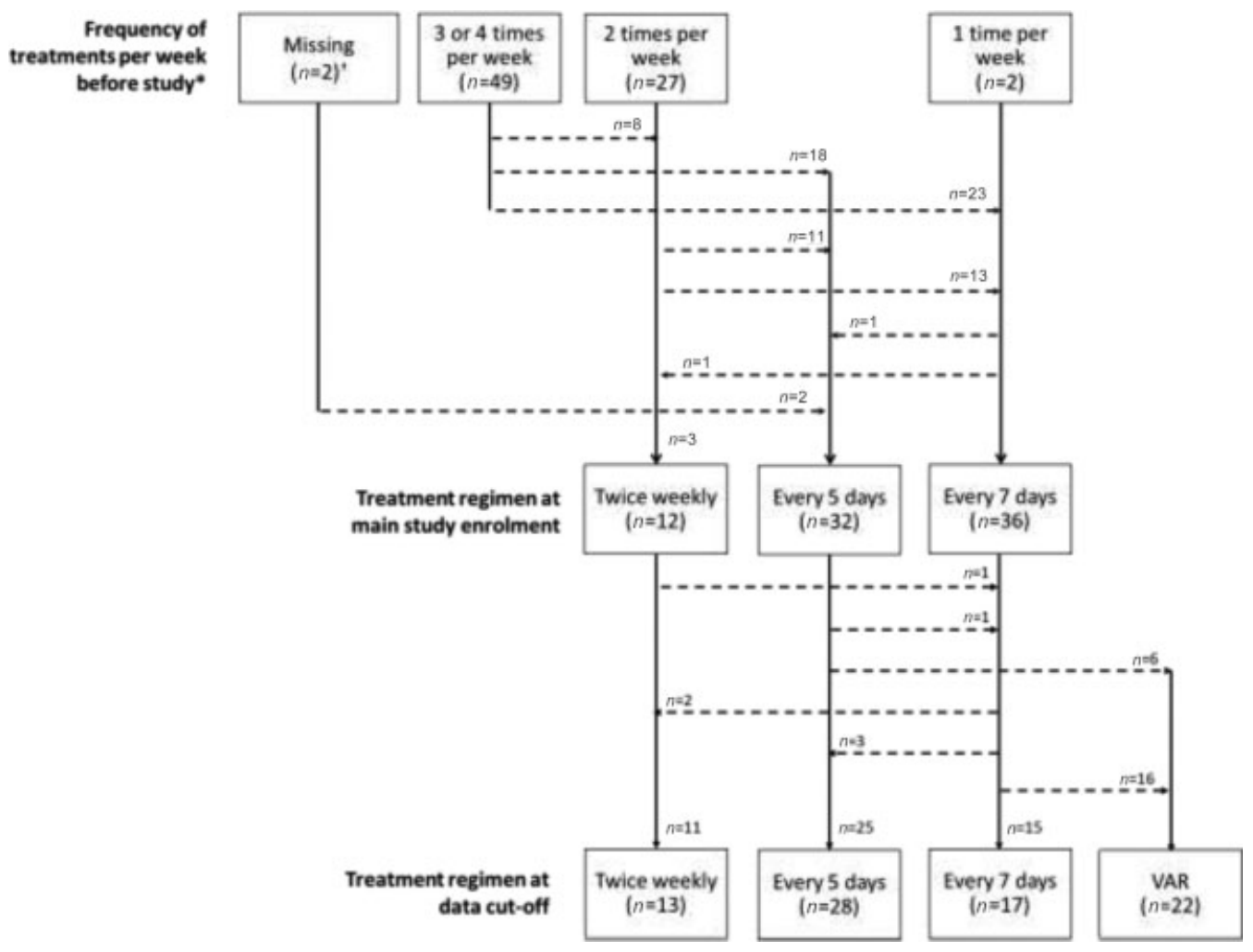

Fig. 2 Previous factor VIII dosing regimen for patients receiving BAY 94-9027 during the PROTECT VIII main study and extension. *Reported by patients in the Treatment Satisfaction and Burden questionnaire at baseline. "Two patients in the "Every 5 days" regimen at data cutoff had an unknown prophylaxis treatment frequency prestudy. One of these patients with unknown prophylaxis treatment frequency also received treatment as "on demand when having a bleed." Variable frequency group included patients who changed treatment group at least once after first infusion beyond 7 days into extension.

transitioning from SHL FVIII products to an EHL rFVIII product, BAY 94-9027. The results demonstrate that, compared with SHL FVIII treatment, BAY 94-9027 prophylaxis offers sustained or improved protection from bleeds with
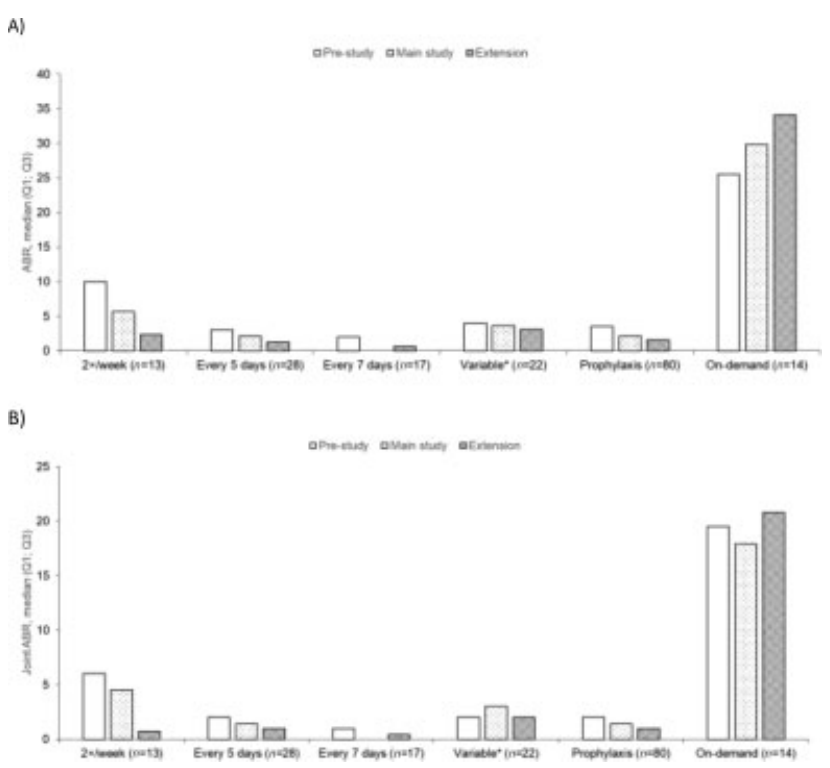

Fig. 3 Median total ABR (A) and joint ABR (B) in BAY 94-9027 prophylaxis patients prestudy and during the PROTECT VIII main study and its extension. ABR, annualized bleed rate; Q, quartile. Treatment regimen based on extension cutoff date (August 2019). less frequent injections. Further improvement in bleeding rates was observed with regular long-term exposure to BAY 94-9027, particularly in the group of patients who were treated twice weekly.

To note, approximately half of the patients who received twice-weekly BAY 94-9027 prophylaxis during the extension were originally assigned to this treatment group because they had a higher bleeding tendency during the run-in phase. ${ }^{13}$ Similarly, the effect of decreasing median total ABR with increasing infusion interval may also be attributed to the patient stratification after the run-in phase. This trend is consistent with the interim analysis of the PROTECT VIII extension study with the full patient cohort. ${ }^{20}$ Patients assigned to the 5- or 7-day infusion interval groups were those who were controlled (defined as not more than one spontaneous bleed) with twice-weekly BAY 94-9027 throughout the 10-week run-in period. Patients who were uncontrolled (defined as two or more spontaneous bleed) during this period remained on twice-weekly prophylaxis, and therefore were excluded from extended-interval prophylaxis.

While at the start of the extension study patients were allowed to switch dosing regimen, most chose to continue with the same regimen. Most patients who switched prophylaxis regimen moved from a lower dosing frequency to a higher dosing frequency, which was due to several reasons including increased bleeding frequency with a lower dosing interval, restarting treatment after injury, and patient 
preference. However, patients who remained in the every-7day group throughout the main and extension studies to date have the lowest median total and joint ABRs in the extension period, compared with all other treatment regimens, which suggests the extended-dosing intervals are effective for some patients. In addition, several patients also switched back to a lower dosing frequency after re-education, or no further increase in bleeding frequency after the first switch. While no formal analysis has been made to assess the impact of switching patients between treatment regimens may have had on the data, the freedom the patients have to switch frequency at any point of the study is a unique study design feature that mimics a real-world clinical setting. During the main and extension studies, the majority of patients were able to extend their dosing interval with BAY 94-9027, with some receiving every-5-day or every-7-day dosing without compromising efficacy with preserved or improved protection from bleeds.

In the present study, patients treated with BAY 94-9027 prophylaxis experienced reduced frequency of joint bleeds compared with patients treated on-demand. In patients with existing joint involvement caused by previous recurrent bleeds, the mechanism underlying joint improvement with the use of regular prophylaxis is likely to be the interruption of the vicious cycle of hemarthrosis-synovitis. ${ }^{21}$ Joints in patients with hemophilia are often inflamed as a consequence of bleeding and are vulnerable to repeated bleeding, perpetuating the damage. ${ }^{22-24}$ Reducing bleeding (including bleeding into target joints) may allow the joints to "cool down" and become less susceptible to further damage, perpetuating a positive-feedback cycle in which less vulnerable joints bleed less, further decreasing observed bleeding rates. ${ }^{22}$ Indeed, a reduction in bleeding rates observed over a longer follow-up period with sustained prophylaxis was observed in the present study, with frequency of joint bleeds also decreasing in the extension period compared with prestudy. At baseline, despite receiving prophylaxis prior to study start, the prevalence of patients in the prophylaxis groups with target joints was high $(72.5 \%)$, and was confirmed by high prestudy ABRs (median ABR [Q1; Q3] = 3.5 $[1.0 ; 10.0]$ in the prophylaxis group). However, joint ABRs decreased across all prophylaxis groups during the main study and extension period of PROTECT VIII, after switching to EHL prophylaxis with BAY 94-9027. Target joint resolution of patients who were previously treated with FVIII prophylaxis prior to entry to PROTECT VIII has been published elsewhere. ${ }^{25}$ Target joints were present in 59 (72\%) out of 82 patients, with a mean (standard deviation) number of historic target joints per patient of 1.4 (1.3) for the whole cohort. Out of a total of 122 historic or new target joints, 111 (91\%) had resolved by the cutoff date.

Data on the effect of switching from SHL to EHL rFVIII products are still limited. However, complementing our findings, a decrease in ABR and joint ABR was observed in patients switching from SHL rFVIII to EHL rFVIIIFc in a singlecenter retrospective study. ${ }^{26}$ This increased protection from bleeds over and above that achieved with SHL rFVIII products constitutes a response to an unmet patient need in hemo- philia A. However, from the patient's perspective, value is more than just efficacy-treatment convenience is key for good adherence with the lifelong infusion schedule. In a survey of more than 1,000 patients with hemophilia (85\% of whom had hemophilia A), efficacy was rated as the second most important attribute for new EHL products, with extended dosing intervals being the most important attribute. $^{27}$

In the present post hoc study, patients transitioned from prophylaxis with SHL FVIII products to prophylaxis with BAY 94-9027, with a marked improvement in ABR compared with prestudy data. Pharmacokinetic studies of BAY 94-9027 demonstrate that it offers a higher AUC compared with SHL rFVIII. ${ }^{12,28}$ This is of interest, as a post hoc analysis of 34 patients showed that higher AUC was associated with reduced bleeding propensity. ${ }^{29}$

This analysis has several limitations. First, the study is limited by its retrospective design. Bleeds were self-reported and, owing to their subjective nature, may be subject to response bias. However, the reliability of the prestudy bleed data was confirmed by the lack of improvement in ABR in the on-demand treatment group. The prestudy mean (median) bleed rate in the 12 months prior to the study in all 14 ondemand patients was 30.1 (25.5) compared with an ABR of 32.5 (29.8) during the main study. In general, patients on FVIII treatment complete patient diaries, which is standard practice in most countries. However, there may be a trend for a lower prestudy bleed rate due to mild bleeding events not being recorded, whereas during a clinical study, all bleeds, including mild bleeds, are more likely to be documented by the patient, depending on the individual's sensitivity. This could be the reason for the slight increase in total ABR in ondemand patients during the prestudy period due to a more stringent reporting of minor bleeds during the PROTECT VIII study compared with the prestudy period, but the clinically relevant joint bleed rate was relatively stable over time. An incomplete documentation of the prestudy bleed rate makes it even more difficult to demonstrate improvement during the study. Third, 29 patients (36\%) included in the prophylaxis group were previously treated with SHL prophylaxis only once or twice a week. This is a lower prophylaxis frequency than the minimum low-dose prophylaxis of two to three times per week with SHL FVIII, as recommended by the World Federation of Hemophilia, ${ }^{1}$ and therefore these patients may have had insufficient prophylaxis prior to study entry. Furthermore, the patients treated on-demand acted as a reliability group, to assess the reliability of the comparison between historic and study data, but no formal comparisons were made.

Evidence following the launch of BAY 94-9027 and from its widespread use in clinical practice outside of clinical trials will provide more information about the effectiveness of this EHL rFVIII with extended dosing intervals compared with patients' previous products. In this respect, HEM-POWR, a multinational, multicenter, noninterventional, open-label, prospective phase 4 cohort study is currently recruiting and, as well as long-term effectiveness, will provide insights into patterns of switching and reasons for regimen/dose 
choice and physical activity and their relationship with clinical outcomes.

\section{What is known about this topic?}

- BAY 94-9027 (damoctocog alfa pegol, Jivi) is a sitespecifically PEGylated, extended-half-life (EHL) B-domain-deleted recombinant factor VIII (rFVIII).

- BAY 94-9027 has an improved pharmacokinetics profile compared with standard-half-life (SHL) rFVIII.

- In the PROTECT VIII study, most patients received BAY 94-9027 every 5 days and had a low annualized bleeding rate.

\section{What does this paper add?}

- Bleed rates and dosing frequency in patients switching from SHL prophylaxis to EHL rFVIII prophylaxis were evaluated.

- Bleeding rates were lower with BAY 94-9027 prophylaxis than with SHL products used prior to enrollment.

Funding

This study was funded by Bayer.

\section{Conflict of Interest}

M.E.M. has received consultancy fees from Bayer Healthcare, CSL Behring, Novo Nordisk, Pfizer, Roche, Sobi, Shire, Octapharma, Kedrion, and Grifols. M.T.R. has received institutional research support from Bayer and BioMarin, and received honoraria for serving on advisory boards and speakers bureaus for Bayer, Novo Nordisk, Sanofi Genzyme, and Takeda. C.N. has received grant/research support, honoraria, or consultation fees from Alnylam, Baxalta/Shire, Bayer, CSL Behring, LFB, Novo Nordisk, Octapharma, Roche, Pfizer, and Sobi. B. A.K. has received research support from CSL Behring and Novo Nordisk, and received honoraria for serving on advisory boards and speaker bureaus from Bayer, BioMarin, CSL Behring, Novo Nordisk, Octapharma, Pfizer, Roche, Sobi, and Takeda. S.R. has received institutional research grants from Sangamo, and participated in speaker bureaus for Shire. M.L.S. has received institutional research grants from Bayer, Bioverativ/Sanofi, Daichii-Sankyo, Octapharma, Novo Nordisk, Baxalta (Shire, Takeda), and Roche (Genentech), and received honoraria and consultation fees from Bayer, BioMarin, Pfizer, Roche, Spark, and Novo Nordisk.

\section{Acknowledgments}

Graeme Baldwin, of Darwin Healthcare Communications, London, England, gave editorial assistance in the writing of this manuscript, fully funded by Bayer. We thank
Monika Maas Enriquez, Maria Wang, and Inga Bayh, of Bayer, for their critical review of this manuscript.

\section{References}

1 Srivastava A, Brewer AK, Mauser-Bunschoten EP, et al;Treatment Guidelines Working Group on Behalf of The World Federation Of Hemophilia. Guidelines for the management of hemophilia. Haemophilia 2013;19(01):e1-e47

2 Gringeri A, Lundin B, von Mackensen S, Mantovani L, Mannucci PMESPRIT Study Group. A randomized clinical trial of prophylaxis in children with hemophilia A (the ESPRIT Study). J Thromb Haemost 2011;9(04):700-710

3 Manco-Johnson MJ, Abshire TC, Shapiro AD, et al. Prophylaxis versus episodic treatment to prevent joint disease in boys with severe hemophilia. N Engl J Med 2007;357(06):535-544

4 Aledort LM, Haschmeyer RH, Pettersson HThe Orthopaedic Outcome Study Group. A longitudinal study of orthopaedic outcomes for severe factor-VIII-deficient haemophiliacs. J Intern Med 1994; 236(04):391-399

5 Tiede A. Half-life extended factor VIII for the treatment of hemophilia A. J Thromb Haemost 2015;13(Suppl 1):S176-S179

6 Björkman S, Folkesson A, Jönsson S. Pharmacokinetics and dose requirements of factor VIII over the age range 3-74 years: a population analysis based on 50 patients with long-term prophylactic treatment for haemophilia A. Eur J Clin Pharmacol 2009;65 (10):989-998

7 Geraghty S, Dunkley T, Harrington C, Lindvall K, Maahs J, Sek J. Practice patterns in haemophilia A therapy - global progress towards optimal care. Haemophilia 2006;12(01):75-81

8 Petrini P, Valentino LA, Gringeri A, Re WM, Ewenstein B. Individualizing prophylaxis in hemophilia: a review. Expert Rev Hematol 2015;8(02):237-246

9 Collins PW, Blanchette VS, Fischer K, et al;rAHF-PFM Study Group. Break-through bleeding in relation to predicted factor VIII levels in patients receiving prophylactic treatment for severe hemophilia A. J Thromb Haemost 2009;7(03):413-420

10 Ar MC, Balkan C, Kavaklı K Extended half-life coagulation factors: a new era in the management of hemophilia patients. Turk J Haematol 2019;36(03):141-154

11 Mei B, Pan C, Jiang H, et al. Rational design of a fully active, longacting PEGylated factor VIII for hemophilia A treatment. Blood 2010;116(02):270-279

12 Coyle TE, Reding MT, Lin JC, Michaels LA, Shah A, Powell J. Phase I study of BAY 94-9027, a PEGylated B-domain-deleted recombinant factor VIII with an extended half-life, in subjects with hemophilia A. J Thromb Haemost 2014;12(04):488-496

13 Reding MT, Ng HJ, Poulsen LH, et al. Safety and efficacy of BAY 949027, a prolonged-half-life factor VIII. J Thromb Haemost 2017;15 (03):411-419

14 Thomson G, et al. Effective protection for $>5$ years with BAY 949027 prophylaxis: interim results from the PROTECT VIII extension trial. Poster Presentations. Haemophilia 2018;24:32-135

15 Reding MT, Ng HJ, Tseneklidou-Stoefer D, Linardi C, Laelzari S. Safety of long-term prophylaxis with BAY 94-9027: interim results of $>5$ years of treatment in the PROTECT VIII extension trial. Haemophilia 2018;24(S5):x

16 European Medicines Agency. JIVI Summary of Product Characteristics. 2018. Accessed January 2019 at: https://www.ema. europa.eu/documents/product-information/jivi-epar-productinformation_en.pdf

17 Pharmaceuticals and Medical Devices Agency. 2018. Available at: https://www.pmda.go.jp/files/000229786.pdf. Accessed January 2021

18 US Food and Drug Administration. JIVI Antihemophilic Factor (Recombinant) PEGylated-aucl Prescribing Information. 2018. Accessed January 2019 at: https://www.fda.gov/downloads/Biologics BloodVaccines/UCM618979.pdf 
19 Batt K, Gao W, Ayyagari R, et al. Matching-adjusted indirect comparisons of annualized bleeding rate and utilization of BAY 94-9027 versus three recombinant factor VIII agents for prophylaxis in patients with severe hemophilia A. J Blood Med 2019;10:147-159

20 Lalezari S, Reding MT, Pabinger I, et al. BAY 94-9027 prophylaxis is efficacious and well tolerated for up to $>5$ years with extended dosing intervals: PROTECT VIII extension interim results. Haemophilia 2019;25(06):1011-1019

21 Valentino LA. Blood-induced joint disease: the pathophysiology of hemophilic arthropathy. J Thromb Haemost 2010;8(09):1895-1902

22 Mulder K, Llinás A. The target joint. Haemophilia 2004;10 (Suppl 4):152-156

23 Aznar JA, García-Dasí M, Pérez-Alenda S, et al. Secondary prophylaxis vs. on-demand treatment to improve quality of life in severe adult haemophilia A patients: a prospective study in a single centre. Vox Sang 2014;106(01):68-74

24 Roosendaal G, Lafeber FP. Pathogenesis of haemophilic arthropathy. Haemophilia 2006;12(Suppl 3):117-121

25 Reding MT, Pabinger I, Lalezari S, Santagostino E, Mancuso ME. Target joint resolution in patients with haemophilia A receiving long-term prophylaxis with BAY 94-9027. Haemophilia 2020;26 (04):e201-e204

26 Wang C, Young G. Clinical use of recombinant factor VIII Fc and recombinant factor IX Fc in patients with haemophilia $\mathrm{A}$ and $\mathrm{B}$. Haemophilia 2018;24(03):414-419

27 von Mackensen S, Kalnins W, Krucker J, et al. Haemophilia patients' unmet needs and their expectations of the new extended half-life factor concentrates. Haemophilia 2017;23(04): 566-574

28 Shah A, Coyle T, Lalezari S, et al. BAY 94-9027, a PEGylated recombinant factor VIII, exhibits a prolonged half-life and higher area under the curve in patients with severe haemophilia A: comprehensive pharmacokinetic assessment from clinical studies. Haemophilia 2018;24(05):733-740

29 Valentino LA, Pipe SW, Collins PW, et al. Association of peak factor VIII levels and area under the curve with bleeding in patients with haemophilia A on every third day pharmacokinetic-guided prophylaxis. Haemophilia 2016;22(04):514-520 